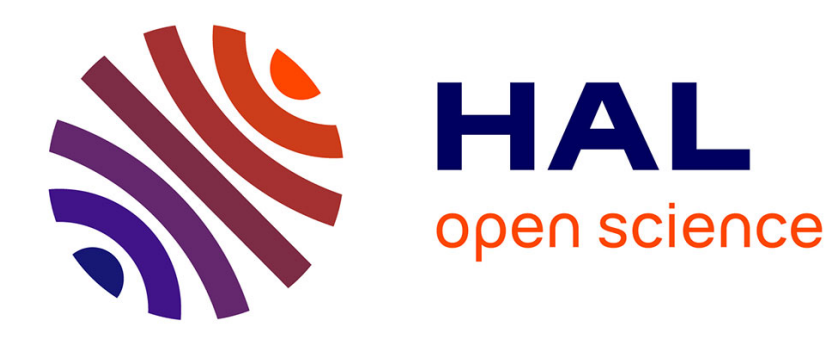

\title{
La référence au futur en basque du nord
}

Georges Rebuschi

\section{To cite this version:}

Georges Rebuschi. La référence au futur en basque du nord. Faits de langues, 2009, Le futur (33), pp.119-132. artxibo-00591751

\section{HAL Id: artxibo-00591751 https://artxiker.ccsd.cnrs.fr/artxibo-00591751}

Submitted on 10 May 2011

HAL is a multi-disciplinary open access archive for the deposit and dissemination of scientific research documents, whether they are published or not. The documents may come from teaching and research institutions in France or abroad, or from public or private research centers.
L'archive ouverte pluridisciplinaire HAL, est destinée au dépôt et à la diffusion de documents scientifiques de niveau recherche, publiés ou non, émanant des établissements d'enseignement et de recherche français ou étrangers, des laboratoires publics ou privés. 


\title{
La référence au futur en basque du nord
}

\author{
Georges Rebuschi*
}

\section{INTRODUCTION}

On va décrire ici les deux principaux moyens de faire référence au futur (qui se définit par rapport au moment d'énonciation), et plus généralement à la postériorité ou ultériorité (i.e. par rapport à un moment quelconque), dans les dialectes basques parlés en France ${ }^{1}$, tels qu'ils sont attestés depuis le milieu du $16^{\mathrm{e}}$ siècle.

L'intérêt typologique de ces données est double. D'abord, dans leur grande majorité, les verbes ne peuvent pas être fléchis directement pour le temps, le mode et l'accord (ce qui est vrai pour tous les dialectes basques) : on a recours à une conjugaison analytique (ou composée), le verbe lexical étant sous forme participiale, et la flexion étant portée par un auxiliaire. Le futur non-marqué est donc une construction analytique, avec un auxiliaire au présent, et un participe "prospectif", qui s'oppose à deux autres participes, l'un accompli, et l'autre inaccompli : il s'agit donc d'un codage aspectuel. Ensuite, et uniquement dans les dialectes cis-pyrénéens, il existe un moyen modal de faire référence au futur, que ce soit par l'ajout d'un suffixe épistémique sur les verbes forts conjugués directement, ou par la même affixation sur les auxiliaires, ce qui laisse la possibilité de moduler aspectuellement le futur, et a permis, à une époque reculée, de construire un "futur dans le futur".

En §2, la morpho-sémantique essentielle de la conjugaison synthétique du basque sera présentée. En $\$ 3$, les formes analytiques seront analysées, dont le "participe futur" ou prospectif, qui entretient une intéressante relation avec l'accompli. En \$4, l'affixe épistémique -ke, évoqué en $\$ 3$, sera repris. Les formes analytiques qui associent les divers participes aspectuels à un auxiliaire doté de ce suffixe seront examinées en $§ 5$. Une récapitulation sera faite en $§ 6$.

\section{LA CONJUGAISON SYNTHETIQUE}

Seuls quelques verbes, dits "forts", et dont le nombre diminue régulièrement depuis cinq siècles, ont une conjugaison synthétique, qui oppose fondamentalement un temps passé à un temps présent. Exemples :

\footnotetext{
* Sorbonne nouvelle \& UMR 7107. Courriel : georges.rebuschi1@free.fr

${ }^{1}$ Soit, d'ouest en est, trois dialectes, le labourdin, le bas-navarrais, et le souletin, mots qui recouvrent chacun des parlers distincts ; noter aussi une influence de plus en plus grande du second dialecte sur le premier, d'où le sous-titre de Lafitte (1962).
} 


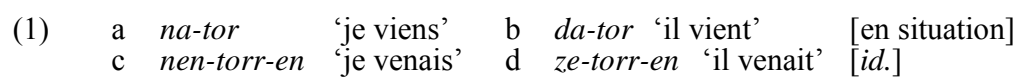

L'opposition entre les temps-accords de $3^{\mathrm{e}} \mathrm{p}$. $d$ - et $z$ - en $(1 \mathrm{~b}, \mathrm{~d})$ marque, pour le second, l'antériorité du repère de l'énoncé (le "point R" de Reichenbach (1947)) par rapport au moment d'énonciation S (pour Speech time chez Reichenbach), et pour le premier, une simple non-antériorité de $\mathrm{R}$ par rapport à $\mathrm{S}$ : comme il n'y a pas de futur non-segmentable qui s'oppose à $d$ - ou $z-$, l'antériorité de $\mathrm{S}$ par rapport à $\mathrm{R}$ et/ou $\mathrm{E}$ (le moment du procès, Event time) est marquée autrement, et c'est bien entendu cela qui fera l'objet essentiel de ce travail.

Noter la redondance de la marque de passé, avec d'un côté un suffixe -(e)n, et, de l'autre, la variation dans les préfixes d'accord: on a le même (?) - $n$ - aux deux premières personnes (sg. comme pl.), et une alternance $d$ - / $z$ - à la troisième.

Il existe enfin une variante "modale" du passé, sans suffixe temporel; les verbes présentent alors les mêmes affixes d'accord qu'au passé pour les deux premières personnes (du singulier et du pluriel), mais ils prennent $l$ - plutôt que $z$ lorsque le préfixe est l'absolutif de $3^{\mathrm{e}}$ personne :

$\begin{array}{llll}\text { (2) (a) } 1 \mathrm{sg} .: \text { nen-tor } & \text { (b) } 3 \mathrm{sg} .: \text { le-tor (ou la-tor) }\end{array}$

De telles formes ne peuvent pas être employées sans affixation supplémentaire ; outre le -en passé, de (1c,d), elles vont par paires :
(2') a ba-nen-tor 'si je venais' [suppositif ou conditionnant] b ba-le-tor 's'il venait' id. c nen-tor-ke 'je viendrais' [conditionnel : conditionné]
d le-tor-ke 'il viendrait' id.

Donc, $b a$ - correspond à 'si' conditionnant, et -ke, dans les formes dites du conditionnel, à la marque de prédiction matérialisée par le - $r$ - du français et le $w$ de will/would (dénué de volition : purement prédictif) de l'anglais.

Associé aux formes du présent et du passé (non modal), -ke apporte, selon les contextes, diverses valeurs épistémiques, de l'équipossibilité à l'expression de la prédiction, en passant par la probabilité. D’où, d'une part, un "potentiel" (présent et passé), reconnu par les grammaires :

$\begin{array}{llll}\text { (3) } & \text { da-tor-ke } & \text { 'il peut venir' } & \text { (cf. (1b)) } \\ \text { b } & \text { ze-tor-ke-en } & \text { 'il pouvait venir' } & \text { (cf. (1d)) }\end{array}$

et, d'autre part, un "futur" qui peut marquer une incontestable certitude de la part du locuteur dans des phrases comme les suivantes (avec le préfixe $d$-du présent, et non le préfixe $l-)$ :

(4) a Baldin hire begia simple bada, si ton œil sain/entier s'-il-est 


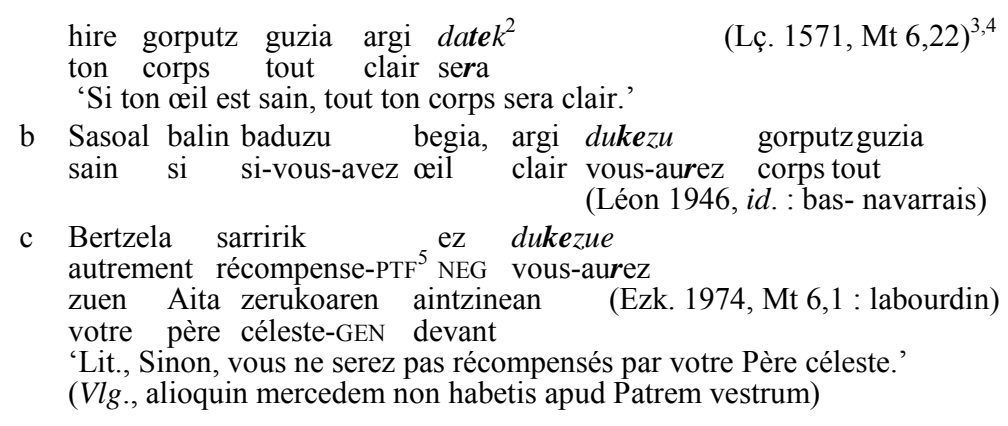

Ce futur modal en -ke est exclusivement employé dans les dialectes parlés en France, et, aujourd'hui, presque uniquement en souletin (Gèze 1873, Gavel \& Lacombe 1937, Coyos 1999) pour les autres dialectes, noter le contexte particulier dans $(4 \mathrm{~b}, \mathrm{c})$ : le $b a$ - de la proposition précédente dans (b) est conditionnant, et bertzela en (c) apporte un présupposé du même type.

Pour revenir au souletin, voici un double exemple (traduction du même texte, de la fin du $19^{\mathrm{e}}$ siècle, puis de la fin du $20^{\mathrm{e}}$ ) dans un contexte non-conditionné maintenant :

Ps $111,6 / 7^{6}$ Vlg., non commovebitur donec despiciat inimicos suos.

a Lotsa gabe da-teke bere etsaiak arrabaskatürik ikhus artio peur sans il-sera POSS ennemis défait-PTF ل voir jusqu'à Lit. 'Il sera sans crainte jusqu'à ce qu'il voie ses ennemis défaits.'

b Bihotza dü-ke bakian eta beldür gabe...

(Ar. 1862)

cœur-SG il-aura paix-LOC et peur sans... Lit. 'Il aura le cœur en paix et sans crainte...'

(C-H 1986, p. 362)

${ }^{2}$ Le verbe 'être', et lui seul, emploie les allomorphes -te et -teke (parfois -teki- devant voyelle) de -ke (voir Lafon 1970-72). Quant au -k final, c'est une marque "allocutive", qui indique une relation (ici) familière, du locuteur à l'interlocuteur: date serait la forme neutre, non-allocutive.

${ }^{3}$ Les références explicites aux sources basques sont données en fin d'article. En ce qui concerne la position dialectale de "Lç."/Leiçarrague, premier traducteur du NT en basque, elle est délicate: le traducteur a manifestement cherché à construire une koinè supradialectale ; la conjugaison de son texte, cependant, correspond plutôt à celle du souletin et des variétés les plus orientales du bas-navarrais (cf. Lafon 1944 : I, p. 60-63).

${ }^{4}$ Les textes basques écrits du $16^{\mathrm{e}}$ au $19^{\mathrm{e}}$ étaient essentiellement religieux, et, de plus, des traductions (Vulgate clémentine, Imitatio de Thomas à Kempis); si les inconvénients sont évidents, mais incontournables, ces textes présentent l'avantage de constituer des paraphrases les uns des autres, ce qui permet de mieux fixer tant la variation dialectale que l'évolution diachronique.

5 Les abréviations suivantes seront utilisées: ACC, accompli; AUX, auxiliaire; COMP, marqueur de subordination; ERG, ergatif; GEN, génitif; INAC, inaccompli; NEG, négation; OD, objet direct; POSS, possessif; PROS, prospectif; PTF, partitif; REL, marqueur de relativation; SG, singulier; VL, verbe lexical; Vlg., Vulgate latine. Le symbole $\sqrt{ }$ devant un verbe indique qu'il s'agit d'un radical, sans flexion (temps/accord), ni suffixe aspectuel. ${ }^{6}$ La numérotation des Psaumes bibliques employée ici est celle de la Vulgate. 


\section{LA PREMIERE CONJUGAISON ANALYTIQUE ET LE FUTUR ASPECTUEL ${ }^{7}$}

\subsection{Les participes associés à un auxiliaire au présent}

La plupart des verbes, on l'a dit, ne peuvent pas être directement fléchis. Ils se présentent donc sous forme participiale, associée à un auxiliaire, soit izan 'être' (avec les verbes intransitifs "inaccusatifs"), soit ukan ' 'avoir' (autres verbes), qui est lui-même fléchi selon les possibilités décrites supra. Les participes, nommés traditionnellement "passé", "présent" et "futur", sont en fait aspectuels. Le premier correspond à un accompli; morphologiquement, la finale de ce participe est variable : par rapport au radical (voir infra), elle peut être $\varnothing,-i,-n$ ou encore $-t u$, seul suffixe productif aujourd'hui. Le second marque l'inaccompli (ou l'itérativité), et se caractérise par une finale en $-t(z) e n$.

Le troisième participe, le plus intéressant de notre point de vue, est le prospectif, morphologiquement dérivé de l'accompli par suffixation de $-k o$ ou -(r)en. Ces deux allomorphes (dont la répartition est partiellement dialectale, et parfois simplement dictée par la finale du participe accompli) renversent l'orientation temporelle entre la borne du procès la plus proche et le repère de l'énoncé (le "point $\mathrm{R}$ " cité supra): la borne droite précède $\mathrm{R}$ dans le cas de l'accompli, et la borne gauche le suit dans le cas du prospectif.

Du côté de l'auxiliaire, on retrouve la tripartition des formes de $3^{\mathrm{e}} \mathrm{p}$. en $d-$, $z$ et $l$ - vue supra. (6) ci-dessous donne les trois combinaisons possibles des participes avec un auxiliaire au présent (ou plutôt au non-passé) :

(6) a sar-tu naiz/da 'je suis / il $1^{9}$ est entré' [na-iz 'je suis'; da 'il est']

b sar-tzen naiz/da 'j'entre/ il entre'

c sar-tu+ko/sar-tu+ren naiz / da 'j'entrerai / il entrera'

(6c) est donc le futur (usuel, non-marqué) de tous les verbes qui ne peuvent se conjuguer directement ${ }^{10}$, mais aussi des verbes forts (sauf en souletin). 7 Pour éviter toute confusion avec des constructions comme celle évoquée dans la note 10 ,
je n'emploierai pas le terme, pourtant bien établi depuis Lafitte (1962), de conjugaison
périphrastique, et emprunterai l'adjectif "analytique" à Oyharçabal (2003), travail que cet
article espère compléter.
${ }^{8}$ Les participes à l'accompli sont employés comme entrées des verbes dans les
dictionnaires. Par ailleurs, si les formes fléchies de ces deux auxiliaires sont toujours
distinctes, la forme izan sert de participe à la fois pour 'être' et pour 'avoir' en labourdin.
9 Il n'y a pas de distinction de genre à la $3^{\mathrm{e}}$ personne : on pourrait aussi bien traduire par
'elle...'. Idem pour les pronoms (ce sont des démonstratifs à la $3^{\mathrm{e}}$ p.).
10 Je n'ai malheureusement pas la place de parler de la périphrase en -t(z)eko plus
auxiliaire, de connotation déontique, identifiée dans Eguzkitza (1978) comme autre forme
de futur (voir aussi Rebuschi (1980) et Coyos (1999)). Ce qui montre que c'est une
périphrase, et non une forme analytique de la conjugaison, c'est (i) que l'OD est
optionnellement au génitif, (ii) que ces formes peuvent être utilisées comme modificateurs
adnominaux, et (iii) qu'elles peuvent s'accorder en nombre avec l'argument à l'absolutif,
ce qui est impossible avec un participe prospectif (ou inaccompli). Noter aussi que -(r)en
n'alterne jamais avec -ko ici, contrairement à ce qui se passe avec le participe prospectif 
Quant à (6b), c'est, pour les verbes faibles, une forme ambiguë entre une interprétation itérative, et une interprétation "progressive", en situation (bien qu'il existe une périphrase, en ari, qui permet de souligner l'inachèvement) mais elle est évidemment purement itérative pour les verbes forts.

\subsection{L'opposition entre futur synthétique modal et futur analytique aspectuel}

Pour les verbes forts, la question se pose de savoir s'il y a une différence sémantique entre les deux futurs, ou bien s'il y en avait une en navarrolabourdin, lorsque les deux futurs coexistaient. Il ne semble pas (même si les occurrences du futur modal en labourdin, quand il était utilisé, i.e. jusqu'à la fin $\mathrm{du} 19^{\mathrm{e}}$ siècle, et en bas-navarrais, sont nettement moindres qu'en souletin). A titre d'exemple, si l'on regarde deux versions des mêmes textes par les mêmes traducteurs, on note la stricte équivalence entre ces deux "conjugaisons". C'est le cas de Mt 18,4 (où le latin offre d'ailleurs un présent dans l'apodose), que l'on trouve d'une part dans la traduction de l'évangile en question, et d'autre part dans celle de l'Imitation de Thomas à Kempis $( \pm 1420: 3,58,9)$ — et ce, tant en souletin, (7), qu'en labourdin, $(8)^{11}$ :

(7) Vlg. \& Thomas, Quicumque ergo humiliaverit se sicut parvulus iste, hic est maior in regno caelorum. 'Quiconque donc se fera humble comme cet enfant, celui-là sera [lit. est] le plus grand dans le royaume...'

a Núr-ere árren txipitüren beita haurtto haur bezala, hura iza-nen da handiena zeliétako erresumán.

b Nur ere arren txipitüren beita haur hau bezala, hura date-ke handiena zelietako erresuman. (Inch., 1883)

(8) a Nork-ere beraz bere burua haurtto hunen heinera baitu aphalduren,

b Zeinak ere beraz hunako haurtto hunen heinera aphalduko baitu bere burua, hura iza-nen da zeruetako erresuman handiena. (Duv., 1896)

Remarquer que le "choix" entre les deux futurs est inversé entre les deux traducteurs: si le souletin offre d'abord (évangile, années 1860) un futur analytique, puis un futur synthétique, c'est le contraire en labourdin, qiui présente un futur synthétique (évangile, mêmes années), puis futur analytique (traduction plus tardive de l'Imitation).

\subsection{Les participes avec un auxiliaire en $\mathbf{z}-$ ou $\mathbf{l}$ -}

Lorsque l'auxiliaire est au passé ( $3^{\mathrm{e}}$ p. $z$-), l'interprétation se complique un peu. Le participe accompli se traduira, selon le contexte, par un passé historique ou un plus-que-parfait: (9a). A l'inaccompli, on a une valeur fondamentalement

stricto sensu. (Pour une brève description de ces formes dans les dialectes d'OutreBidassoa, voir Hualde (2003), qui les distingue aussi de la conjugaison analytique.)

${ }_{11}$ Faute de place, je ne peux donner le mot-à-mot, mais les formes respectivement synthétiques (dateke, daite) et la forme analytique (izanen da) sont reconnaissables. 
itérative (cas non-marqué dans les phrases indépendantes), mais elle peut également être "progressive", en particulier dans les subordonnées temporelles: (9b). Enfin, au prospectif, si la valeur naturelle de la combinaison est celle d'une visée depuis un repère $\mathrm{R}$ antérieur à $\mathrm{S}$, c'est aussi (devenu) la construction la plus usuelle pour un conditionnel passé (et donc contrefactuel), cf. (9c-ii).

$$
\begin{array}{ll}
\text { a sar-tu nintzen / zen, } & \text { 'j'étais / il était entré '//'j'entrai/ il entra' } \\
\text { b sar-tzen nintzen(ean) / zen(ean) '(quand) j'entrais / il entrait' } \\
\text { c sar-tuko/sar-turen zen } & \text { (i) 'il allait entrer' / (ii) 'il serait entré' }
\end{array}
$$

Une troisième valeur pour $(9 \mathrm{c})$ résulte d'un futur analytique comme celui de (6c), translaté dans le passé au discours indirect ; la forme ne se distingue alors de $(9 \mathrm{c})$ que par l'affixe de subordination -(e)la (qui "absorbe" toujours celui, en $-(e) n$, du passé) :

(10) a Erran du/zuen : 'Sartuko naiz'. 'Il a dit : "J'entrerai.".',
b Erran du/zuen sartuko zela. 'Il a dit qu'il entrerait.'

En ce qui concerne les combinaisons des participes avec un auxiliaire de $3^{\mathrm{e}} \mathrm{p}$. en $l$-, considérons d'abord les formes des protases (en $b a-$ 'si') $)^{12}$. Les plus usuelles sont en les suivantes :

(11) a sar-tu ba-litz / ikusi ba-lu 's'il était entré'/ 's'il l'avait vu.' b sar-tzen ba-litz / ikusten ba-lu 's'il entrait' / 's'il le voyait'

L'association d'un participe prospectif avec de telles formes auxiliaires pour renvoyer à un procès hypothétique futur, donc possible, plutôt que présent, donc contrefactuel, n'est pas rare, mais il s'y ajoute souvent une modalité déontique :

(12) sar-tu+ko ba-litz 's'il devait entrer'.

Par contre, dans les apodoses, au non-passé, c'est le participe prospectif qui est le plus ordinairement employé, associé à un auxiliaire toujours pourvu du suffixe modal -ke, qui indique bien à nouveau une forme de prédiction :

(13) sar-tu+ko liza-te(ke) / ikus-i+ko lu-ke 'il entrerait' / 'il (le) verrait.'

Au passé (contrefactuel), on l'a dit, c'est la forme illustrée en $(9 \mathrm{c})$ qui est la plus usitée, bien qu'elle soit anormale du point de vue compositionnel. (Avec un verbe transitif, on aurait par ex. ikusiko zuen 'il l'aurait vu'). Mais deux autres formes sont à noter. La première, (14), très vivante jusqu'au $17^{\mathrm{e}}$ siècle, et recommandée aujourd'hui par l'Académie basque, se construit en ajoutant -ke à l'auxiliaire; la seconde, (15), dans laquelle le participe reste à l'accompli, semble être sortie d'usage au début du $20^{\mathrm{e}}$ siècle.

(14) Sar-tu+ko zate(ke)en / ikus-i+ko zukeen'il serait entré' / 'il l'aurait vu'

${ }^{12}$ D'autres formes peuvent être construites, avec les auxiliaires de $2^{\mathrm{e}}$ série, cf. $\S 4$. 
(15) Sar-tu liza-te(ke) / ikus-i luke (id.) $)^{13}$

Dans (16) et (17), (Ps 54,12) la construction non-marquée, (9c), et la forme plus rare de type (15) alternent; il est notable que les deux traducteurs (Archu, comme Inchauspé, était souletin, et Duvoisin, labourdin) ont inversé leur choix, ce qui montre à nouveau l'équivalence sémantique des deux constructions ${ }^{14}$ :

(16)

$\begin{array}{lllll}\text { a Ene } & \text { etsaiak gaitzetsitü } & \text { banündü, } & \text { enizün } \\ \text { mon } & \text { ennemi maudit-ACC } & \text { hitzik } \\ \text { s'il-m'avait } & \text { NEG+j'avais } & \text { mot-PTF } \\ \text { ere } & \text { erra-n+en; } & & \\ \text { même } & \text { dire-PROS }\end{array}$

Lit., 'Si mon ennemi m'avait maudit, je n'aurais même pas dit (un) mot.'

b Hügüntzen nündienak nitzaz gaizki handi erran balü, haïr-INAC AUX-REL-SG-ERG sur-moi mal grand dit s'il-l'avait beharbada gorde nündükezün harenganik. (Ar.,1862) peut-être caché-ACC je-m'étais-ke ${ }^{16}$ de-lui

Lit., 'Si celui qui me haïssait avait dit grand mal de moi, je me serais peut-être gardé/caché de lui.'

(17) a Baldin ene etsaiak madarikatu $\operatorname{izan}^{17}$ banindu,

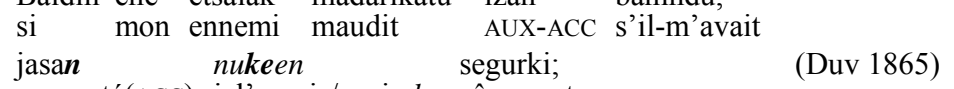
supporté(ACC) jel'aurais/avais-ke sûrement

$\mathrm{b}$ eta gaitzirizkotan nindaukana, ene kontra goraki et en-aversion celui-qui-me-tenait contre moi à-voix-haute mintzatu izan balitz, gordeko nintzen ... haren ganik. parlé(ACC) AUX(ACC) s'il-était, cacher-PRos je-m'étais de lui

En résumé, outre le glissement des formes conditionnelles au prospectif vers une valeur modale en (12), il y a toujours eu, dans le basque attesté, un jeu d'échange, voire de superposition, entre le marqueur d'aspect prospectif -ko/-en et le modal -ke, ce qui semble indiquer un équilibre instable entre une de référence faite à un moment ultérieur par rapport à un repère donné (prospectif), et l'explicitation d'une prédiction ou visée (affixe -ke) à partir de la situation non-réelle construite dans et par la protase conditionnelle.

${ }^{13}$ La récapitulation des "systèmes éventuels" dans Lafitte $(1962: \S 720)$ indique pourtant la seconde comme fréquente, mais ne mentionne même pas la première, citée comme potentielle dans des tableaux plus détaillés à la fin de l'ouvrage.

${ }^{14} \mathrm{Vlg}$. pour (16-17): Quoniam si inimicus meus maledixisset mihi, sustinuissem utique. Et si is qui oderat me super me magna locutus fuisset, abscondissem me forsitan ab eo.

${ }^{15}$ Forme allocutive polie, souletine, au passé (labourdin neutre : ez nuen).

${ }^{16}$ L'auxiliaire est à nouveau allocutif (poli) : c'est ici l'équivalent d'être' donnant une valeur médio-passive ou réfléchie pour le prédicat.

${ }_{17}$ On a ici, et dans la phrase suivante, une forme "surcomposée", avec, après le participe lexical accompli, une forme participiale de l'auxiliaire également à l'accompli (izan équivanelent de ukan), puis enfin l'aux. fléchi. Ces formes sont fréquentes dans les hypothétiques passés. 


\section{LA DEUXIEME CONJUGAISON ANALYTIQUE ET LE SUFFIXE - KE}

Il existe une seconde série d'auxiliaires, défectifs (ils n'ont pas de formes nonfléchies), qui s'associent au radical des verbes lexicaux; leur racine est respectivement $-(d) i$ pour les verbes inaccusatifs, et $-z a$ - pour les transitifs et assimilés Le procès est alors saisi globalement (aspect "aoristique" dans la terminologie d'A. Culioli). On obtient ainsi, entre autres, des subjonctifs, par ex. sar dadin 'qu'il entre' ikus dezan 'qu'il (le) voie' (dans une proposition régie par un verbe au présent), sar zedin (régissant au passé, et concordance des "temps"),

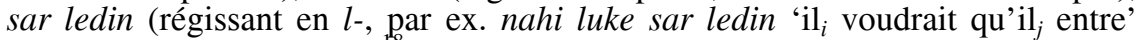
[sujets non-coréférentiels]) ${ }^{18}$.

En ce qui nous concerne, c'est le potentiel qui est important, parce qu'il mobilise le mophème -ke :

$\begin{array}{llll}\text { (18) } & \text { a sar dai-te(ke) / ikus deza-ke 'il peut entrer / (le) voir' } \\ & \text { b sar z(a)i-teke-en / ikus zeza-ke-en } & \text { 'il pouvait entrer / (le) voir' }\end{array}$

Quand le préfixe de $3^{\mathrm{e}} \mathrm{p}$. est $l$-, on obtient de nouvelles formes hypothétiques éventuelles, i.e. non-contrefactuelles, mais -ke conserve (plus ou moins) la valeur de potentiel qu'il acquiert quand il est affixé à un auxiliaire de la seconde série :

(19) Sar ba-ledi, ikus leza-ke 's'il entrait, il le verrait/pourrait le voir.'

Les verbes forts, quand ils sont fléchis directement, correspondent dans certains contextes (i) soit à un véritable progressif : erraiten dautzut datorrela 'je vous dit qu'il vient/est en train de venir', cf. ... etortzen (inac.) dela '... qu'il vient (régulièrement)', soit (ii) à une forme aoristique : manatu dut datorrela, cf. ... etor (radical) dadila 'j'ai ordonné qu'il vienne', paraphrase construite avec un auxiliaire de seconde série. Il s'ensuit que la suffixation de -ke sur un verbe lexical fléchi est naturellement ambiguë: elle peut s'interpréter comme un potentiel (le verbe lexical étant interprété comme en (ii)), soit comme une marque de probabilité et/ou prédiction, d'où sa spécialisation comme marqueur de futur en souletin, et ses multiples valeurs en labourdin, surtout ancien.

\section{PARTICIPES ASPECTUELS ASSOCIES A UN AUXILIAIRE EN - KE}

Examinons à présent ce qui se passe lorsque -ke est suffixé à un auxiliaire combiné à un participe marqué aspectuellement.

\subsection{Le "futur antérieur" ou accompli}

Il se construit évidemment avec un participe accompli et un aux. en -ke au présent. On le trouve le plus souvent dans des circonstancielles temporelles, dans des contextes où il fonctionne comme terme marqué par rapport à une

${ }^{18}$ Chez Lç. (1571), sar zedin était un pur passé aoristique, le subjonctif étant toujours en l-. 
circonstancielle au futur analytique ordinaire. Comme dans le cas du futur synthétique (en -ke), c'est dans les textes souletins qu'on en trouve le plus grand nombre d'occurrences. Les trois versions de (20) sont ainsi assez représentatives, (a) étant en souletin, et (b) en bas-navarrais et en labourdin - (c), labourdin également, donnant la paraphrase avec le futur synthétique du passage :

(20) Mt 24,15, Vlg., Cum ergo videritis abominationem desolationis... 'Quand vous aurez vu l'abomination de la désolation...'
(Duv. 1865, Léon 1946)
(Hrt. 1855)

Mais on trouve également des formes surcomposées, comme en (21a), en labourdin, en face de la version (b), autre traduction souletine :
(21) Jn 21,18, Vlg., cum autem senueris...

Enfin, on trouve aussi le participe inaccompli dans des contextes qui conduisent pourant le lecteur à ordonner chronologiquement le procès de la circonstancielle d'abord, puis celui de l'apodose. Le futur antérieur latin de Mt 24,15, cf. (20), est ainsi rendu de cette manière par Inchauspé (1856) : Ikhus-ten düzi-enian, lit. 'quand vous le voyez'.

Pour le même "temps" latin en Mt $2,8, \ldots$ et cum inveneritis, renuntiate mihi... 'et quand vous l'aurez vu, avertissez-moi...', on trouve :

- un futur antérieur, de type (20a), dans tous les dialectes et à toutes les époques : dans Lç. (1571), Haran. (1740), Inch. (1856), Duv. (1865) et Léon (1946);

- un présent inaccompli, comme en $(6 \mathrm{~b})$, dans Iribarnegaray $( \pm 1860)$ : basnavarrais occidental, et Cazenave $( \pm 1860)$ : bas-navarrais oriental ${ }^{21}$;

\footnotetext{
${ }^{19}$ Jusqu'au $18^{\mathrm{e}}$ siècle, on peut aussi trouver le subjonctif; pour ce passage, c'est le cas dans Lç. (1571): çahar adinean (forme tutoyée), et Hrt. (1855) : zahar zaitezenean (forme vouvoyée); noter l'absence de suffixe aspectuel, et l'auxiliaire de seconde série, en - $a(d) i-$. Remarquer aussi que le -te- du dernier aux. n'est pas un allormorphe de -ke ici, mais une marque de pluriel purement morphologique, liée au vouvoiement.

${ }^{20} \mathrm{La}$ fermeture $\mathrm{du} / e / \mathrm{de}-k e$ - devant une autre voyelle est fréquente (cf. la note 2 ).

${ }^{21}$ On trouve aussi un inaccompli + auxiliaire sans -ke chez ces deux traducteurs pour Mt 21,40 (Cum ergo venerit dominus vineae, quid faciet agricolis illis?) : resp. ji-ten de-laik et ji-ten de-nian, lit. 'venant il-est- quand...', soit 'quand il vient...' ; et des exemples du même type en souletin, cf. (28) plus loin dans le texte : cet emploi semble donc être, ou avoir été, principalement oriental, ce qui reste cependant à vérifier de plus près.
} 
- un futur analytique ordinaire, Ezk. $(1974)^{22}$.

Pour Jn 7,31 (Vlg., Christus cum venerit, numquid plura signa faciet, 'Quand le Christ viendra, fera-t-il plus de miracles...?'), on a la répartition suivante :

- le futur antérieur: Duv. (1865), labourdin : ethorr-i di-teke-nean; Ur. (1873), souletin : ji-n da-teki-enean ;

- le présent synthétique, dathorrenean, cheiz Lç. (1571);

-1 ' inaccompli + auxiliaire au présent simple : Haran. (1740), labourdin ;

- le prospectif + aux. au présent simple : Léon (1946), bas-navarrais, et Ezk. (1974), labourdin.

On le voit, si la langue donne bien le moyen de marquer explicitement l'antériorité d'un événement futur par rapport à un autre, elle n'y oblige pas le locuteur - et ce, peut-être d'autant plus qu'une interprétation épistémique de probabilité est toujours disponible pour la combinaison accompli + aux. en $-k e$ : de même qu'en français il l'au $r_{1} r_{2} a_{3} v_{4} u_{5}$ peut s'interpréter aussi bien comme l'équivalent de il $a_{1+2} d_{2}-\hat{u}_{5}$ le voi ${ }_{4} r$ (jugement de probabilité), que comme un "vrai" futur antérieur, de même, en basque, dans une phrase indépendante, $i k u s_{4}-i_{5} \quad d_{3}-u_{1}-k e_{2}$ a les deux interprétations: ce n'est que dans les circonstancielles que la valeur de futur antérieur, ou "passé dans le futur", se comprend automatiquement.

\subsection{Accompli et parfait résultatif}

Superficiellement, l'accompli s'oppose à une forme voisine, le parfait résultatif, dans laquelle, en navarro-labourdin, le participe s'accorde en nombre avec l'OD quand le verbe est transitif, et avec le sujet quand il est intransitif (inaccusatif); en souletin, le participe accompli est alors au partitif, -( $r) i k$. La différence sémantique est usuellement nette : dans le premier cas, le procès est simplement présenté comme arrivé à son terme, alors que dans le second, c'est l'état résultant du référent de l'argument accordé, ou parfois de l'univers de discours, qui est dénoté, cf. (22)-(23) :

a Haurrek $\begin{array}{lll}\text { ogia jan } & \text { dute. } \\ \text { enfants-ERG } & \text { pain-SG } & \text { mangé(ACC) } \\ \text { ils-l'on }\end{array}$

'Les enfants ont mangé le pain.' (ils ont fini de manger)

b Haurrek ogia jan-a dute.

enfants-ERG pain-SG mangé(ACC)-SG ils-l'ont

'Les enfants ont mangé le pain.' (il n'y en a plus)

(23) a Haurra erori da

'L'enfant est tombé.' (événement)

b Haurra erori-a da

enfant-SG tombé(ACC)-SG il-est

'L'enfant est tombé.' (il est à terre)

22 On peut aussi trouver des futurs antérieurs dans ce texte presque contemporain, par ex. pour Mt 18,15 (Si te audierit, lucratus es fratrem tuum) : ontsa hartzen bazitu, zure aneia irabazi dukezu, lit. 'S'il vous reçoit bien, vous aurez gagné votre frère'. 
Ce parfait résultatif ${ }^{23}$ a de plus en plus concurrencé le futur accompli, ailleurs qu'en souletin :

(24) Mt 6,16, Vlg., Receperunt mercedem suam. 'ils ont (déjà) reçu leur récompense.'

$\begin{array}{lll}\text { izatu }^{24} \text { dukete-la hek bere saria. (Haran. 1740) } & \end{array}$ avoir-ACC ils-auront-COMP eux POSS salaire

Lit. '(je vous dis) qu'ils ont eu/reçu leur prix/récompense

(25)- a izan-a dute hek bere saria (Hrt 1855)

$\mathrm{b}$ ukan-a dutela beren saria. (Ezk. 1974)

Il en va évidemment de même avec des prédicats intransitifs - (26a) est en souletin, et (b), en labourdin :

(26) Ps 48,17, Vlg., Ne timueris cum dives factus fuerit homo.

a Ez beldürt gizon bat aberats-tü datenian

NÉG Vcraindre homme un enrichi-ACC quand-il-sera

'N'aie pas peur quand un homme s'est enrichi'

b Ez lotsa, gizona aberas-tu-a denean NEG Vcraindre homme-sG enrichi-sG quand-il-(s')est

(Duv. 1865)

\subsection{L'inacccompli avec auxiliaire au futur en -ke}

Les participes inaccomplis peuvent aussi, beaucoup plus rarement, s'associer à un auxiliaire au futur (en -ke). Outre les ex. bas-navarrais signalés en 5.1, qui ne correspondent pas à la valeur fondamentale de l'inaccompli, on en trouve quelques exemples en souletin, dialecte dans lequel cette valeur est bien réelle :

(27) Vlg. 48,11, Non videbit interitum, cum viderit sapientes morientes.

Eztü ikhus-iko heriua, zühürrak hil-tzen ${ }^{25}$

NEG+AUX voir-PROS mort-SG sages mourant(INAC)

ikhus-ten düz-ki-enian

voir-INACC AUX-ke-REL-LOC

'Il ne verra pas la mort quand il verra [= en voyant] les sages mourir.'

Ici, la valeur imperfective est claire, mais ce n'est pas le cas dans $(28)^{26}$ :

${ }^{23}$ Qui, associé à la "mise au chômage" ou à la suppression de l'agent des constructions transitives, a donné lieu à l'émergence d'un passif périphrastique.

${ }^{24}$ Variante de izan.

${ }^{25}$ Cette forme n'est pas un part. inaccompli, mais un gérondif (homophone) régi par le verbe de perception de la matrice (ikusi 'voir/vu'). Elle s'en distingue par le fait que lorsque le verbe est transitif, l'OD peut ou doit (selon les dialectes) se mettre au génitif.

${ }^{26}$ La plupart des autres traductions consultées donnent un futur composé non-marqué, ethorriko denean, de Harriet (1855) à Ezkila (1974), en passant par Duvoisin (1860) et Léon (1946). Leiçarrague (1571), quant à lui, a un présent synthétique : Dathorrenean... (cf. (20c) pour un ex. du même type trois siècles plus tard). 
(28) Vlg., Cum ergo venerit dominus vineae, quid faciet agricolis illis? Ji-ten datekienian mahastiaren nausia, venir-INACC sera-REL-LOC vigne-SG-GEN maitre zer eginen dü mahasti-zaiñ haier? quoi faire-PROS AUX vigneron DEM-PL-DATIF

'Quand le propriétaire de la vigne reviendra, que fera-t-il à ces vignerons ?'

De tels exemples n'étaient pas rares, et Gavel \& Lacombe (1937: 56) indiquaient encore dans une note que la forme composée ikusten duke ('voir' inac. + aux. présent en -ke) est "un des équivalents sémantiques du futur simple" [i.e. du futur analytique non-marqué ikusiko du $]^{27}$. Cependant, cela ne semble plus être le cas de nos jours: selon Coyos (1999: 245), une forme comme hartzen düke s'interprète (comme dans les autres dialectes) comme exprimant la probabilité : 'il doit (peut-être) prendre' - mais il est possible que le fait que cette combinaison apparaisse soit dans une proposition indépendante, soit dans une circonstancielle, joue un rôle ici.

\subsection{Le futur du ou dans le futur}

Divers philosophes ont remarqué que la combinatoire de trois fois trois références "temporelles" : præsens, præteritum et futurum situées in prcesenti, in prceterito et in futuro donnait neuf possibilités, dont toutes n'étaient pas, ou que très rarement, matérisalisées dans les langues naturelles; voir par ex. Gardies (1975:146-147) à propos des de la remarque sur les "temps composés dans le sens" de la Grammaire de Port-Royal :

"Arnauld et Lancelot ont remarqué qu'il existait, en français, au moins une possibilité inexploitée, celle d'"ajouter un quatrième temps composé, savoir, celui qui eût marqué l'avenir avec rapport au présent", c'est-à-dire qui eût désigné ce qui sera présent par rapport à l'avenir [...]. Le souci d'être complet eût exigé qu'on signalât encore deux autres possibilités inexploitées : à savoir l'indication de ce qui était futur par rapport au passé, et celle de ce qui sera futur par rapport au futur. Mais nos langues laissent ici des vides [...]"28.

C'est le "futur par rapport au futur" (e.g. latin scripturus ero) qui nous intéresse $\mathrm{ici}^{29}$. En fait, la possibilité offerte de combiner participe prospectif et auxiliaire au futur (i.e. pourvu de -ke) donne naturellement, quand l'aux. est fléchi au présent, un "futur dans le futur". Or avec cette valeur sémantique, la combinaison en question est effectivement attestée au $16^{\mathrm{e}}$ siècle. Dans l'extrait

${ }^{27}$ Gèze (1873 : 197) donnait aussi comme équivalentes les combinaisons esken-tü+ren düt et esken-tzen düket 'je (l')offrirai'.

${ }^{28}$ C'est moi, G.R., qui souligne. Du côté des grammairiens et linguistes, par contre, se reporter à Jespersen ([1924] $1971: 359$ sv.) qui renvoie à un travail bien antérieur (1914) dans lequel il critiquait déjà cette conception des neuf "temps" composés "pour le sens" chez Madvig (un philologue danois mort en 1886) et quelques autres...

${ }^{29}$ Cette forme est prise en compte dans certains travaux contemporains de logiciens, par ex., entres autres, Areces \& Blackburn (2005). 
suivant du $N T$, on peut même apprécier la succession iconique d'un futur antérieur (qui apparaît deux fois : uka-n dukeenari, begira-tu ditukeenari), d'un futur non-marqué (ema-n+en draukat), et d'un futur ultérieur (goberna-tu+ren dituke) :

(29) Apoc 2, 26-27, Vlg., Et qui vicerit, et custodierit usque in finem opera mea, dabo illi potestatem super gentes, et reget eas in virga ferrea...

Biktoria ukan dukeenari $[\alpha]$, eta ene

victoire avoir/obtenir-ACC il-l'aura-REL-SG-DATIF et POSS1SG

obrak finerano begiratu ditukeenari[ $\left.\alpha^{\prime}\right]$,

œuvres fin-jusqu'à observé-ACC il-les-aura-REL-SG-DATIF

emanen draukat $[\beta]$ bothere Jentilen gainean.

donner-PROS je-le-lui-PRESENT pouvoir Gentils-GEN sur

Eta gobernaturen dituke $[\gamma]$ burdinazko zihorrez...

et gouverner-PROS il-les-aura de-fer verge-INST

Lit. 'A celui qui aura $[\alpha]$ la victoire et qui observera[ $\alpha$ '] mes œuvres jusqu'à la fin, je donnerai $[\beta]$ le pouvoir sur les Gentils, et il les gouvernera $[\gamma]$ avec une verge de fer. ${ }^{30}$

Aucune des traductions ultérieures ne présente de forme équivalente à celle de $[\gamma]$ dans ce passage, pas même Haraneder (1740) qui, pourtant, emploie cette combinaison assez fréquemment, mais sans qu'on puisse y déceler une différence de valeur par rapport au futur composé ordinaire de type $[\beta]$, comme par ex. dans sa traduction de (28) supra:

(30) Ethorriko ditekenean, beraz, mahastiaren jabea... venir-PROS AUX-ke-REL-LOC donc vigne-SG-GEN propriétaire-SG 'Quand viendra donc le propriétaire de la vigne...'

\section{CONCLUSION}

Nous avons été amenés à distinguer, du côté morpho-syntaxique, entre la conjugaison synthétique (réservée aux quelques verbes dits forts), et la conjugaison composée ou analytique (où le participe du verbe lexical est associé à un auxiliaire fléchi). Dans le premier cas, les verbes forts peuvent prendre le suffixe épistémique -ke, qui, selon le contexte, s'interprète comme codant l'équipossibilité, la probabilité, ou la prédiction — d'où un futur "synthétique", mais segmental ou agglutinatif, encore très vivant en souletin. Les autres verbes ne peuvent qu'apparaître sous forme participiale (ou radicale), associés à un auxiliaire fléchi. L'un de ces participes est clairement prospectif : il indique que la borne gauche du procès se situe dans un segment temporel postérieur au repère de l'énoncé. Mais comme les auxiliaires sont nécessairement des verbes forts, ils peuvent eux-mêmes prendre le suffixe modal $-k e$, d'où un grand nombre

${ }^{30}$ Liç. (Mt 9,15) présente un exemple aussi clair, avec un premier futur, non-marqué, suivi d'un futur dans le futur, pour renvoyer à un second événement, qui ne peut se produire que postérieurement au premier. 
de combinaisons a priori possibles dans un métasystème qui n'a peut-être jamais été totalement exploité, mais qui a pu offrir des nuances importantes, dont un "futur du futur", disparu depuis le $18^{\mathrm{e}}$ siècle, et un "présent" (i.e. un inaccompli) du futur, qui ne survit que comme équivalent, dans les circonstancielles, du futur périphrastique ordinaire.

\section{SOURCES BASQUES}

Nota. Les références suivies d'un astérisque sont accessibles sous forme $\mathrm{rtf}$ sur le site : $\mathrm{http} / / /$ klasikoak.armiarma.com/alfa.htm.

Celles suivies d'undouble astérisque sont accessibles sous forme photographique sur le site : http://www.euskadi.net/LiburutegiDigitala/

[Ar.] Archü, J.-B., 1862, Daviden gorantzak edo psalmiak.*

[C-H] Casenave-Harigile, J., 1986, Egün oroetako irakurgeiak, Saint-Sébastien, Itxaropena.

Cazenave, A., \pm 1860 , Jesu Khristoin Ebanjelio Seindia San Mathiü̈n aäbera, ms. publié par R. M. Pagola et al. (eds.), Bonaparte Ondareko Eskuizkribuak, Ekialdeko Behenafarrera, vol. I, Bilbao, Deustuko Unibertsitatea, 1999, p. 17-76.

[Duv.] Duvoisin, J.-P, 1865, Bible edo Testament Zahar eta Berria [...].**

----- Duvoisin, J.-P, 1896, Jesu-Kristoren Imitazionea, P. Haristoy Ziburuko errotaraz argitara emana. [L'Imitation de J.-C., publiée par P.H., recteur de Ciboure]** [accessible sous l'entrée Haristoy]

[Ezk.] (anon.), 1974, Jesu Kristoren Berri Ona. Bonloc (Pyrénées Atlantiques), Editions Ezkila.

[Haran.] Haraneder, J. 1740, Jesu Christoren Evangelio Saindua.*

[Hrt.] Harriet, M.,1855, Iesu-Christo gure Iaunaren Testament Berria [...]. Bayonne, Lasserre.

[Inch.] Inchauspé, E., 1856, Le saint Evangile selon saint Mathieu, traduit en basque souletin, Bayonne, Lamaignière.

----- Inchauspé, E., 1883, Jesu-Kristen Imitacionia.**

Iribarnegaray, X., \pm 1860 , Yesu Kristoin Ebanjeliua San Mathioin arabera, ms. ed. par R. M. Pagola et al., dans Bonaparte Ondareko Eskuizkribuak, Mendebaldeko behenafarrera, Bilbao, Deustuko Unibertsitatea,1999, p. 17-81.

[Lç.] Leiçarrague, J., 1571, Iesus Christ Gure Iaunaren Testamentu Berria [...].*

Léon, L., 1929, Jesu-Kristoren Imitazionea.*

Léon, L., 1946, Jesu-Kristo gure Jaunaren Ebanjelio Saindua, Ustaritz, publié par l'auteur.

[Ur.] Urruthy, A., 1873, Ebanjelio Saintia Jesus-Kristena Jondane Johaneren arabera.** 